\title{
Body composition in asymptomatic HIV-infected men: cross-sectional and prospective assessment
}

\author{
Marios C Lazanas ${ }^{1}$, Irene V Lambrinoudaki ${ }^{2}$, Gerasimos A Douskas ${ }^{2}$, \\ Georgios A Tsekes ${ }^{2},{ }^{1}$ Maria N Chini ${ }^{1}$, Evangelos K Georgiou ${ }^{2}$
}

${ }^{1}$ Second Department of Internal Medicine - AIDS Unit, Tzanion Hospital, Piraeus, Greece, ${ }^{2}$ Department of Medical Physics, Athens University Medical School, Athens, Greece

\begin{abstract}
Data on body composition changes in HIV infected patients is sparse and controversial. The aim of this study was to assess body composition in asymptomatic HIV-infected men with normal body weight in comparison to healthy HIV-negative control men and to investigate possible body composition changes in HIV-positive patients over a 2-year observation period. One hundred eight asymptomatic seropositive men, aged 19-62 years, and 20 healthy sex, age and weight - matched controls were recruited for the cross-sectional part of the study. Fifty-eight of the HIV+ patients were followed up for 2 years. Body weight, BMI, Bone Mineral Content (BMC), body Fat mass (Fat), \% Fat, body Lean mass (Lean) and \% Lean was recorded for each subject at the beginning and at the end of the follow-up period. The same analysis was repeated separately for arms, trunk and legs. HIV+ men had increased fat mass and reduced lean mass compared to controls $(\%$ Fat in HIV +24.3 , \%Fat in controls 19.2, $p=0.012$; \% Lean in HIV + 72.1, \%Lean in controls 77.0, $p=0.014)$. Lean mass was lower in extremities while fat mass was higher in the trunk region in HIV+ in comparison to controls, irrespective of antiretroviral therapy. Longitudinally, patients with higher baseline \%Fat (>24.2, median) presented $20 \%$ decrease in fat mass while patients with lower baseline \%Fat $(<=24.2)$ showed a smaller, non-significant decrease in fat mass accompanied by a significant decrease $(\mathbf{2 . 5 2 \%})$ in lean mass. Fat loss occurred in all subjects predominantly in the extremities (16.5-36.45\% loss), with relative preservation of trunk fat. It is concluded that otherwise asymptomatic HIV + men exhibit subtle body composition changes involving reduced lean mass and increased central fat mass. The pattern of weight loss over time depends on baseline fat store: patients with adequate fat stores lose predominantly fat while patients with lower baseline fat stores lose both fat and lean mass. In the entire cohort, there is a tendency towards central adiposity, with the majority of fat being lost from the extremities, a picture resembling metabolic $x$ syndrome.
\end{abstract}

Key words: DEXA. body composition, HIV

Address correspondence and requests for reprints to:

Dr. Irene Lambrinoudaki, MD, 18 Kifissias Str, GR-115 26, Athens, Greece, Telephone and FAX: +30-210-7759569,

e-mail: ilambrinoudaki@hotmail.com

Received 02-10-02, Revised 08-11-02, Accepted 13-12-02 


\section{INTRODUCTION}

Body wasting is a major feature of the AIDS syndrome. The severity of wasting in HIV infected patients and especially the degree of body cell mass depletion is closely related to survival ${ }^{1}$. Moreover, weight reduction in the asymptomatic HIV-positive subject seems to be a predisposing factor for the progression to the AIDS syndrome ${ }^{2}$. The process of weight loss over the course of HIV infection is not yet fully clarified. It is suggested that weight loss in HIV-positive patients results from a cachectic response to the disease and consists predominantly of lean tissue loss with relative preservation of fat tissue ${ }^{3-6}$. On the other hand, some investigators report that weight loss is merely a process of fat loss as a result of reduced energy intake or increased energy expenditure ${ }^{7-10}$. Most studies addressing the issue of body composition in HIV-infected patients are cross-sectional in design while only a few describe body composition changes in HIV-positive subjects prospectively. Moreover, the variety of methods of body composition assessment employed contributed further to the controversy of the results ${ }^{6,11}$.

The aim of this study was to assess first on a crosssectional basis body composition parameters in HIVpositive asymptomatic men in comparison to sex, age and weight matched healthy controls and second to investigate body composition changes in the HIV-positive individuals over a study period of two years.

\section{SUBJECTS AND METHODS}

\section{Subjects}

One hundred eight HIV infected, asymptomatic men aged $19-62$ years were studied. HIV + men were recruited from the AIDS unit of the $2^{\text {nd }}$ Department of Internal Medicine, Tzanion Hospital, Piraeus, Greece. HIV infection was diagnosed on the basis of a positive western blot for serum HIV-1 antibodies. All subjects were in good clinical condition and had a normal body weight. Twenty healthy male volunteers, HIV negative, matched for age and body weight, were recruited from the hospital staff and served as controls. All subjects gave informed consent and the study was approved by the Hospital Ethical Committee.

\section{Study protocol}

Patients and controls were subjected to baseline evaluation in the morning after consumption of a light breakfast. After a thorough physical examination, subjects were weighed with light clothing on an electronic balance. Subsequently, body composition analysis was performed by whole body Dual Energy X-ray Absorptiometry (DEXA). The following parameters were estimated: Bone Mineral Content (BMC), Fat tissue mass (Fat) and fat-free soft tissue mass (Lean). The same parameters were also estimated separately for arms, trunk and legs.

The second follow-up evaluation was performed 2 years after the baseline visit. From the 108 HIV-positive patients assessed at baseline, 58 were recruited for follow-up, 5 of them being on antiretroviral treatment. No difference was found between responders to our call and non-responders with respect to baseline demographic characteristics and body composition analysis parameters.

\section{Measurements}

Body composition measurements were performed by use of a Hologic QDR-2000 machine. The principles of the procedure have been described elsewhere ${ }^{12,13}$. Data acquisition has been made in fan beam mode. The rationale of the technique is based on the attenuation of $\mathrm{x}$-ray beams through the body. The attenuation of $\mathrm{x}$-rays by a subject depends on the subject mass and composition. X-rays of different energies are attenuated to different extent by fat and fatfree tissues. When two x-ray energies are used, two tissue components can be determined at any one time. When bone is not present, the ratio of the attenuation is linearly related to the proportion of fat in the soft tissue. Thus, by measuring this attenuation ratio, the proportion of fat and fat-free tissue can be determined. The tissue composition in terms of fat and fatfree mass in the regions where bone is detected is extrapolated from the surrounding areas where no bone is detected. Thus, DEXA can measure three tissue components: BMC, Fat mass and Lean mass.

\section{Statistical analysis}

The statistical package SPSS was used for data analysis (Statistical Package for the Social Sciences, Chicago, Illinois, Version 6.0). Mean values of baseline parameters were compared between patients and controls by Student's t-test for unpaired observations. Mean follow-up values were compared to baseline by Student's t-test for paired observations. Statistical significance was set at the 0.05 level. 


\section{Results}

Mean baseline characteristics and whole body composition analysis in HIV-positive and control men are presented in Table 1. No difference was found, as expected, between patients and controls with respect to age, body weight and BMI. BMC and Lean mass were lower in the patients compared to the control group. Conversely, \% Fat mass was higher in the patients in comparison to controls.

Mean baseline regional body composition in HIVpositive and control men is presented in Table 2. BMC was lower in all regions in HIV-positive subjects compared to controls. Fat mass in the trunk was significantly higher in patients, compared to controls. On the other hand, Lean mass in the extremities was significantly lower in the patients in comparison to the controls. The same results were obtained when patients on antiretroviral or protease inhibitor therapy were excluded from the analysis. Neither CD4 count nor HIV seropositivity duration was correlated with any of body composition parameters.

Baseline and follow-up whole body composition analysis in the 58 HIV-positive patients who completed the study period is shown in Table 3. Analysis was performed separately in two groups: in patients with baseline $\%$ whole body fat mass $\leq 24.2$ (median,

Table 1. Baseline characteristics and whole body composition analysis [Mean (SD)] in 108, clinically stable, non-wasted HIV - positive men and 20 healthy HIV-negative age and weight-matched control men.

\begin{tabular}{lccc}
\hline & HIV+ & Control & P* \\
\hline Age & $37.8(8.8)$ & $35.2(8.8)$ & NS \\
Weight & $74.1(12.1)$ & $75.2(9.0)$ & NS \\
BMI & $24.3(3.5)$ & $23.6(2.8)$ & NS \\
CD4 count & $380,9(224,8)$ & - & \\
Months since HIV & & & \\
seropositivity diagnosis & $57.3(42.5)$ & - & \\
Antiretroviral therapy N (\%) & $11(10.2 \%)$ & - & \\
Bone Mineral Content (Kg) & $2.55(0.39)$ & $2.83(0.33)$ & 0.003 \\
Fat mass (Kg) & $18.0(7.2)$ & $14.6(7.7)$ & 0.056 \\
Fat mass (\% body weight) & $24.3(8.2)$ & $19.2(8.8)$ & 0.012 \\
Lean mass (Kg) & $52.3(8.6)$ & $57.7(8.6)$ & 0.012 \\
Lean mass (\% body weight) & $72.1(7.9)$ & $77.0(8.5)$ & 0.014 \\
\hline
\end{tabular}

* Student's t-test for unpaired observations
$\mathrm{N}=29$ ) and in patients with baseline \% whole body fat mass $>24.2(\mathrm{~N}=29)$. BMC declined significantly in both groups over the 2-year period. A statistically significant decrease in Lean mass was observed in the first group while Fat mass showed a non-significant decrease. By contrast, a marked reduction in Fat mass was observed in the second group with a small but statistically significant increase in Lean mass.

Baseline and follow-up regional body composition analysis, separately for patients with baseline $\%$ whole body fat mass lower and higher than 24.2 (median) are presented in Table 4. In the first group, Lean mass decreased predominantly in the trunk region while no significant change was observed in the extremities. On the other hand, Fat mass decreased in the extremities of the same group with a non-significant increase in the trunk. In the second group, fat decreased predominantly in the extremities with a parallel small increase in Lean mass. Overall, the exclusion of the five patients on antiretroviral treatment in the follow-up visit did not modify our results.

Table 2. Baseline regional body composition analysis [Mean (SD)] in 108 clinically stable non-wasted HIV - positive men and 20 healthy HIV-negative, age and weight matched control men.

\begin{tabular}{|c|c|c|c|}
\hline & HIV+ & Controls & $\mathbf{P}^{*}$ \\
\hline \multicolumn{4}{|l|}{ Arms } \\
\hline Bone Mineral Content (Kg) & $0.40(0.08)$ & $0.47(0.08)$ & 0.001 \\
\hline Fat mass $(\mathrm{Kg})$ & $2.35(1.06)$ & $2.32(1.82)$ & NS \\
\hline Fat mass ( $\%$ body weight) & $26.8(11.3)$ & $21.5(11.8)$ & 0.055 \\
\hline Lean mass (Kg) & $6.01(1.51)$ & $7.57(1.61)$ & 0.0001 \\
\hline Lean mass (\% body weight) & $68.6(11.1)$ & $73.9(11.3)$ & 0.05 \\
\hline \multicolumn{4}{|l|}{ Trunk } \\
\hline Bone Mineral Content (Kg) & $0.63(0.11)$ & $0.72(0.11)$ & 0.002 \\
\hline Fat mass $(\mathrm{Kg})$ & $8.31(4.44)$ & $4.73(4.05)$ & 0.001 \\
\hline Fat mass ( $\%$ body weight) & $22.5(9.8)$ & $13.7(9.7)$ & 0.0001 \\
\hline Lean mass (Kg) & $26.48(4.01)$ & $28.03(4.31)$ & NS \\
\hline Lean mass ( $\%$ body weight) & $75.7(9.6)$ & $84.2(9.5)$ & 0.0001 \\
\hline \multicolumn{4}{|l|}{ Legs } \\
\hline Bone Mineral Content (Kg) & $1.00(0.17)$ & $1.12(0.16)$ & 0.002 \\
\hline Fat mass $(\mathrm{Kg})$ & $6.40(2.49)$ & $6.61(2.39)$ & NS \\
\hline Fat mass ( $\%$ body weight) & $26.9(9.2)$ & $25.0(8.4)$ & NS \\
\hline Lean mass (Kg) & $16.21(3.13)$ & $18.54(2.81)$ & 0.002 \\
\hline Lean mass (\% body weight) & $68.9(8.9)$ & $70.7(8.0)$ & NS \\
\hline
\end{tabular}

*Student's t-test for unpaired observations 
Table 3. Baseline and follow-up whole body composition analysis [Mean (SD)] in 58 HIV - positive men who completed the 2-year follow-up period.

\begin{tabular}{|c|c|c|c|c|}
\hline & Baseline & 2 year follow-up & $\%$ change from baseline & $\mathbf{P}^{*}$ \\
\hline \multicolumn{5}{|c|}{ Baseline $\%$ body fat $\leq 24.2, \mathrm{~N}=29$} \\
\hline Weight (Kg) & $70.8(10.2)$ & $67.8(8.4)$ & $-3.79(6.02)$ & 0.002 \\
\hline Bone Mineral Content $(\mathrm{Kg})$ & $2.61(0.35)$ & $2.48(0.36)$ & $-5.00(3.31)$ & 0.0001 \\
\hline Fat mass (Kg) & $12.24(3.91)$ & $11.13(3.36)$ & $-5.29(22.7)$ & NS \\
\hline Lean mass $(\mathrm{Kg})$ & $55.15(7.57)$ & $53.73(7.39)$ & $-2.52(3.75)$ & 0.001 \\
\hline \multicolumn{5}{|c|}{ Baseline $\%$ body fat $>24.2, \mathrm{~N}=29$} \\
\hline Weight (Kg) & $78.6(11.2)$ & $74.5(11.1)$ & $-5.10(5.94)$ & 0.0001 \\
\hline Bone Mineral Content (Kg) & $2.62(0.41)$ & $2.53(0.42)$ & $-3.15(4.34)$ & 0.0001 \\
\hline Fat mass $(\mathrm{Kg})$ & $23.63(5.10)$ & $19.10(7.30)$ & $-20.74(3.84)$ & 0.0001 \\
\hline Lean mass $(\mathrm{Kg})$ & $51.55(7.84)$ & $52.86(7.32)$ & $2.81(4.95)$ & 0.013 \\
\hline
\end{tabular}

*Student's t-test for paired observations

Table 4. Baseline and follow-up regional body composition analysis [Mean (SD)] in 58 HIV - positive men who completed the 2-year followup period.

\begin{tabular}{|c|c|c|c|c|c|}
\hline & & Baseline & 2 year follow-up & $\%$ change from baseline & P* \\
\hline \multicolumn{6}{|c|}{ Baseline $\%$ body fat $\leq 24.2, N=29$} \\
\hline \multirow[t]{3}{*}{ Arms } & Bone Mineral Content (Kg) & $0.42(0.07)$ & $0.40(0.06)$ & $-4.05(6.15)$ & 0.001 \\
\hline & Fat mass (Kg) & $1.57(0.46)$ & $1.26(0.37)$ & $-16.50(22.6)$ & 0.0001 \\
\hline & Lean mass $(\mathrm{Kg})$ & $6.84(1.31)$ & $6.78(1.22)$ & $-0.35(8.70)$ & NS \\
\hline \multirow[t]{3}{*}{ Trunk } & Bone Mineral Content $(\mathrm{Kg})$ & $0.65(0.09)$ & $0.60(0.11)$ & $-6.85(8.70)$ & 0.0001 \\
\hline & Fat mass $(\mathrm{Kg})$ & $5.07(2.73)$ & $5.42(2.72)$ & $19.01(41.32)$ & NS \\
\hline & Lean mass $(\mathrm{Kg})$ & $27.73(3.59)$ & $26.52(3.25)$ & $-4.13(5.77)$ & 0.001 \\
\hline \multirow[t]{3}{*}{ Legs } & Bone Mineral Content $(\mathrm{Kg})$ & $1.01(0.15)$ & $0.95(0.15)$ & $-5.70(4.46)$ & 0.0001 \\
\hline & Fat mass $(\mathrm{Kg})$ & $4.68(1.50)$ & $3.52(1.39)$ & $-22.61(23.38)$ & 0.0001 \\
\hline & Lean mass $(\mathrm{Kg})$ & $16.92(2.96)$ & $17.02(3.11)$ & $0.55(5.94)$ & NS \\
\hline \multicolumn{6}{|c|}{ Baseline $\%$ body fat $>24.2, \mathrm{~N}=29$} \\
\hline \multirow[t]{3}{*}{ Arms } & Bone Mineral Content $(\mathrm{Kg})$ & $0.41(0.09)$ & $0.39(0.08)$ & $-5.35(8.74)$ & 0.001 \\
\hline & Fat mass $(\mathrm{Kg})$ & $3.13(0.88)$ & $2.31(1.19)$ & $-28.03(24.58)$ & 0.0001 \\
\hline & Lean mass $(\mathrm{Kg})$ & $5.79(1.41)$ & $6.07(1.27)$ & $6.34(11.68)$ & 0.023 \\
\hline \multirow[t]{3}{*}{ Trunk } & Bone Mineral Content $(\mathrm{Kg})$ & $0.65(0.12)$ & $0.64(0.12)$ & $-1.13(6.92)$ & NS \\
\hline & Fat mass $(\mathrm{Kg})$ & $11.37(3.33)$ & $10.51(4.07)$ & $-7.97(23.35)$ & NS \\
\hline & Lean mass (Kg) & $26.03(3.51)$ & $26.56(3.64)$ & $2.20(6.50)$ & NS \\
\hline \multirow[t]{3}{*}{ Legs } & Bone Mineral Content $(\mathrm{Kg})$ & $1.03(0.17)$ & $0.98(0.18)$ & $-4.92(5.71)$ & 0.0001 \\
\hline & Fat mass (Kg) & $8.14(1.98)$ & $5.25(2.68)$ & $-36.45(24.26)$ & 0.0001 \\
\hline & Lean mass $(\mathrm{Kg})$ & $15.95(2.83)$ & $16.60(2.61)$ & $4.45(6.87)$ & 0.003 \\
\hline
\end{tabular}

*Student's t-test for paired observations

\section{Discussion}

HIV-infected, asymptomatic men in our study had lower lean tissue mass and higher fat mass compared to sex, age and body weight-matched healthy HIV negative controls. The main difference in fat content between patients and controls derived from the trunk while the respective difference in lean mass derived from the extremities. During the 2-year follow-up 
period, two patterns of body composition changes became apparent, depending on the baseline fat content: patients with higher body fat content lost predominantly fat, mainly from the extremities. By contrast, patients with lower body fat content lost both fat and lean mass, the latter being statistically significant.

Previous studies showed that HIV-infected patients with advanced disease had reduced body fat as well as reduced body lean mass ${ }^{12,14}$. The magnitude of tissue depletion has been related to death irrespective of the cause of wasting ${ }^{15}$. Patients with more advanced disease stage, as reflected by CD4 count, had lower body fat mass ${ }^{9,16}$. The reduction in lean body mass, however, has been observed in patients in earlier clinical stages with higher CD4 counts as well ${ }^{16}$. Asymptomatic HIV-infected patients with normal body weight had reduced body cell mass and a trend towards increased body fat mass in comparison to healthy controls ${ }^{5}$. A more recent, large cross-sectional study, conducted in HIV-infected and healthy controls, revealed that HIVinfected men weighed less than their sex-matched controls and that fat-free mass accounted for $51 \%$ of the difference in weight ${ }^{3}$.

Data on the changes in body composition in HIVinfected patients over the course of the disease are more sparse. Asymptomatic seropositive men who finally progress to AIDS exhibit a significant decrease in all body compartments, in association with an increase in resting energy expenditure and fat oxidation, suggesting a cachectic host response ${ }^{2}$. In another longitudinal study in AIDS patients, weight loss was accounted for primarily by fat-free mass as assessed by a variety of methods ${ }^{6}$. During a two-year follow-up period, $31 \%$ of 187 HIV-positive men exhibited significant body cell mass depletion and $12 \%$ had an additional $5 \%$ body cell mass loss in the subsequent 6month interval, which was associated with fatigue, distress and depressive symptomatology ${ }^{4}$. A small, prospective study on body composition changes in HIVinfected men revealed, in concordance with our results, that body composition changes in HIV-positive men over time depend on baseline body fat content: patients with higher body fat exhibit predominantly fat loss while patients with lower body fat content show mainly lean loss ${ }^{1}$.

The use of antiretroviral therapy (reverse transcriptase or protease inhibitor therapies) in HIV-in- fected patients has been increasingly associated with body composition changes, mainly a redistribution of body fat to the trunk region ${ }^{17-19}$. In our study, only a small proportion of HIV-positive patients were on such therapy. Statistical analysis revealed the same results when these patients were excluded from the dataset, implying that body composition changes observed in this study occurred independently of antiretroviral therapy.

The pathogenetic mechanisms involved in body composition changes in HIV-positive subjects have not been elucidated. Reduced caloric intake or inadequate nutrition seems not to play a causative role ${ }^{2}$. Increased resting energy expenditure as a feature of HIV infection has been reported by various authors ${ }^{20-22}$ already in the asymptomatic stage of the disease ${ }^{10}$. De novo hepatic lipogenesis and serum triglycerides were found increased in asymptomatic HIV-infected patients with normal body weight ${ }^{23}$. More recently, a syndrome of peripheral insulin resistance and hypertriglyceridemia was recognized in HIV-infected patients, independent of antriretroviral therapy ${ }^{24}$. Hyperinsulinemia in these patients was highly correlated with reduced body lean mass. Furthermore, one may consider that the emerging picture resembles the metabolic $\mathrm{x}$ syndrome with insulin resistance as the predominant feature ${ }^{25,26}$. Reduced gonadal function and reduced responsiveness of the GH - IGF-I axis have been implicated for the metabolic changes and body composition alterations observed in these patients ${ }^{24,27}$. Testosterone therapy has been reported to restore lean body mass and thus improve insulin sensitivity ${ }^{24}$. Finally, increased 24-hour cortisol excretion by HIV-positive subjects has been linked to the abnormal trunk fat accumulation, possibly as an effect of endogenous hypercortisolism, probably reflecting increased production of cortisol or peripheral cortisol resistance ${ }^{3}$

In conclusion, HIV-positive men exhibit lower body lean mass and higher \% fat mass in comparison to sex, age and weight-matched controls, even in the early asymptomatic stage with normal body weight. Longitudinally, subjects with adequate fat stores seem to lose predominantly fat while subjects with lower fat stores lose both lean and fat mass. The loss of fat mass seems to occur mainly in the extremities, indicating the trend seen in HIV-infected patients towards central fat accumulation in the course of the disease. Larger studies of longer duration are needed to confirm 
our results.

\section{Acknowledgement}

This work has been sponsored by the Greek National Scholarship Foundation IKY (Scholarship Nr $144 / 3^{\text {rd }}$ post-doctoral programme 1999-2000).

\section{REFERENCES}

1. Mulligan K, Tai VW, Schambelan M, 1997 Cross-sectional and longitudinal evaluation of body composition in men with HIV infection. J Acquir Immune Defic Syndr Hum Retrovirol 15: 43-48.

2. Sharpstone D, Murray C, Ross H, et al, 1999 The influence of nutritional and metabolic status on progression from asymptomatic HIV infection to AIDS-defining diagnosis. AIDS 13: 1221-1226.

3. Kotler DP, Thea DM, Heo M, et al, 1999 Relative influences of sex, race, environment, and HIV infection on body composition in adults. Am J Clin Nutr 69: 432-439.

4. Wagner GJ, Ferrando SJ, Rabkin JG, 2000 Psychological and physical health correlates of body cell mass depletion among HIV+ men. J Psychosom Res 49: 55-57.

5. Ott M, Lembcke B, Fischer H, et al, 1993 Early changes of body composition in human immunodeficiency virus infected patients: tetrapolar body impedance analysis indicates significant malnutrition. Am J Clin Nutr 57: 15-19.

6. Paton NI, Macallan DC, Jebb SA, 1997 Longitudinal changes in body composition measured with a variety of methods in patients with AIDS. J Acquir Immune Defic Syndr Hum Retrovirol 14: 119-127.

7. Macallan DC, Noble C, Baldwin C, 1995 Energy expenditure and wasting in human immunodeficiency virus infection. N Engl J Med 333: 83-88.

8. Suettmann U, Ockenga L, Hoogestraat L, et al, 1993 Resting energy expenditure and weight loss in human immunodeficiency virus - infected patients. Metabolism 42: 11731179.

9. Forrester J, Spiegelman D, Woods M, et al, 2001 Weight and body composition in a cohort of HIV-positive men and women. Public Health Nutr 4: 743-747.

10. Sharpstone DR, Murray CP, Ross HM, 1996 Energy balance in asymptomatic HIV infection. AIDS 10: 1377-1384.

11. Corcoran C, Anderson EJ, Burrows B, et al, 2000 Comparison of total body potassium with other techniques for measuring lean body mass in men and women with AIDS wasting. Am J Clin Nutr 72: 1053-1058.

12. Slosman DO, Casez JP, Pichard C, et al, 1992 Assessment of whole body composition with dual-energy X-ray absorptiometry. Radiology 185: 593-598.

13. Drinkwater DT, Bailley DA, Faulkner RA, et al, 1993 Soft tissue analysis by DXA: comparison of QDR-1000W and
QDR-2000 scanners. J Bone Miner Res 8: 352.

14. Kotler DP, Wang J, Pierson MS, 1985 Body composition studies in patients with the aqscuired immunodeficiency syndrome. Am J Clin Nutr 42: 1255-1265.

15. Kotler DP, Tierney AR, Wang J, et al, 1989 Magnitude of body-cell-mass depletion and the timing of death from wasting in AIDS. Am J Clin Nutr 50: 444-447.

16. Grady C, Ropka M, Anderson R, et al, 1996 Body composition in clinically stable men with HIV infection. $\mathrm{J}$ Assoc Nurses AIDS Care 7: 29-38.

17. Mulligan K, Tai VW, Algren H, et al, 2001 Altered fat distribution in HIV-positive men on nucleoside analog reverse transcriptase inhibitor therapy. J Acquired Immune Defic Syndr 26: 443-448.

18. Lo JC, Mulligan K, Tai VW, et al, 1998 "Buffalo hump" in men with HIV-1 infection. Lancet 351: 867-870.

19. Carr A, Samaras K, Burton S, et al, 1998 A syndrome of peripheral lipodystrophy, hyperlipidemia and insulin resistance in patients receiving HIV protease inhibitors. AIDS 12: 51-58.

20. Hommes MJT, Romijn JA, Endert E, et al, 1991 Resting energy expenditure and substrate oxidation in human immunodeficiency virus (HIV)-infected asymptomatic men: HIV affects host metabolism in the early asymptomatic stage. Am J Clin Nutr 54: 311-315.

21. Grunfeld C, Pang M, Shimmizu L, et al, 1992 Resting energy expenditure, caloric intake, and short-term weight change in human immunodeficiency syndrome. Am J Clin Nutr 55: 455-460.

22. Schwenk A, Hoffer-Belitz E, Jung B, et al, 1996 Resting energy expenditure, weight loss and altered body composition in HIV infection. Nutrition 12: 595-601.

23. Hellerstein MK, Grunfeld C, Wu K, et al, 1993 Increased de novo hepatic lipogenesis in human immunodeficiency virus infection. J Clin Endocrinol Metab 76: 559-565.

24. Hadigan C, Corcoran C, Stanley T, et al, 2000 Fasting hyperinsulinemia in human immunodeficiency virus - infected men: relationship to body composition, gonadal function and protease inhibitor use. J Clin Endocrinol Metab 85: 35-41.

25. Chen D, Misra A, Garg A, 2002 Lipodystrophy in human immunodeficiency virus - infected patients. J Clin Endcorinol Metab 87: 4845-4856.

26. Mynarcik DC, Combs T, McNurlan MA, Scherer PE, Komaroff E, Gelato MC, 2002 Adiponectin and leptin levels in HIV - infected subjects with insulin resistance and body fat redistribution. J Acquir Immune Defic Syndr 31: 514-520.

27. Grinspoon S, Corcoran C, Lee K, et al, 1996 Loss of lean body and muscle mass correlates with androgen levels in hypogonadal men with acquired immunodeficiency syndrome and wasting. J Clin Endocrinol Metab 81: 40514058. 